\title{
Associations of post-migration dietary and physical activity behaviours with acculturation and social cognitive factors: a cross-sectional study of Australian residents born in sub-Saharan Africa
}

\author{
Isaac Yeboah Addo ${ }^{1 *}$ (D) Loren Brener ${ }^{1}$, Augustine Danso Asante ${ }^{2}$ and John de Wit ${ }^{1,3}$
}

\begin{abstract}
A considerable amount of research suggests that several 'immigrants' from low-and-middle income countries often adopt less healthy dietary and physical activity behaviours after settling in high income countries, which may lead to increased risk of weight-related diseases. Several

studies have also reported that post-migration changes in dietary and physical activity behaviours are associated with acculturation. Given that social cognitive factors are proximal determinants of behaviour, understanding their interplay with acculturation in the process leading to less healthy weight-related behaviours can assist in developing more useful interventions for populations at risk. A cross-sectional survey was undertaken among Australian residents born in sub-Saharan Africa (SSA) to assess the interplay of post-migration dietary and physical activity behaviours with acculturation and social cognitive factors. A total of 253 participants were self-selected from two Australian states: New South Wales and Victoria. Theory of planned behaviour variables were employed as social cognitive factors. Fat intake and fruits/vegetables/fibre intake were used as indicators of dietary behaviour. Acculturation was measured using two sub-scales: cultural maintenance and cultural participation. The findings show that acculturation and social cognitive factors were significantly associated with variances in fat intake and physical activity. More specifically, the variance in post-migration fat intake was significantly explained by cultural participation and attitude while the variance in physical activity was significantly associated with cultural maintenance and behavioural intention. It is therefore important to consider both acculturation and social cognitive factors when developing weight-related interventions for Australian residents born in SSA.
\end{abstract}

Keywords: Social cognitive factors, Acculturation, Dietary and physical activity behaviours, Australian residents of sub-Saharan African ancestry, Migration

\section{Introduction}

Migration and less healthy changes in dietary and physical activity behaviours

Several studies have reported that migration from a low to a higher income country often leads to less healthy changes in dietary and physical activity behaviours $[1,2]$. For example, a significant increase in fat intake was

\footnotetext{
* Correspondence: i.addo@unsw.edu.au; yebaddo9@yahoo.com ${ }^{1}$ Centre for Social Research in Health, Faculty of Arts and Social Sciences, University of New South Wales, Sydney 2052, Australia

Full list of author information is available at the end of the article
}

found in a systematic review of studies of European residents who emigrated from low-income countries [2]. In the USA, a study of residents who emigrated from Gulf countries noted a significant decrease in physical activity and a low intake of fruits/vegetables after immigration [3]. There is evidence to also suggest that these less healthy dietary and physical activity behaviours which often occur after immigration may put immigrants at risk of weight-related diseases, such as diabetes, some cancers, cardiovascular diseases, and chronic respiratory diseases [4]. 


\section{Australian residents born in sub-Saharan Africa}

In 2014, a screening project organised by the Western Melbourne Regional Development Australia noted that $68 \%$ of Australian residents of African ancestry were overweight, obese, or morbidly obese, which was higher than the national average of $61.3 \%$ [5]. Findings from several previous studies indicate that post-migration changes in dietary and physical activity behaviours may contribute to these weight-related issues $[1,6,7]$. However, there is a dearth of research examining the factors associated with changes in dietary and physical activity behaviours among Australian residents born in sub-Saharan Africa (SSA).

\section{Factors associated with less healthy changes in dietary and physical activity behaviours}

Given that less healthy changes in dietary and physical activity behaviours have negative health implications for immigrants [4], it is important to understand the factors that influence post-migration dietary and physical activity behaviours. Previous studies have attributed post-migration changes in dietary and physical activity behaviours to acculturation [1-3]. Acculturation may be defined as "a dual process of cultural and psychological change that takes place as a result of contact between two or more cultural groups and their individual members" [8]. A number of acculturation theorists have suggested that various behaviour trajectories can emerge after people migrate from their own countries of birth to new countries and these behaviour trajectories can be categorised into two broad aspects: cultural maintenance (i.e. keeping one's own culture after getting in contact with other dominant culture(s)) and/or cultural participation (i.e. adopting the mainstream culture of the host country at the expense of one's own culture) [9-11]. It is however believed that the tendency for a person to maintain his/her own culture and/or adopt the culture of a different group of people is often influenced by group-level factors (i.e. broad social, cultural, economic, environmental and structural factors) $[8,12]$. For instance, a group of migrants from SSA can replace their familiar traditional food by new food options in a Western country, due to limited availability of the familiar food in the host country. On an individual-level, social cognitive factors (e.g. a person's intention and attitude), which are theorised extensively as proximal determinants of behaviour, may mediate the influence of acculturation on post-migration behaviours [1315]. Despite the importance of social cognitive factors in understanding the processes leading to behaviour patterns, little empirical research has tested their interplay with acculturation in understanding post-migration dietary and physical activity behaviours.

\section{Theory of planned behaviour}

Theory of planned behaviour, which is a widely known social cognitive theory, has been employed in this study to help explain variations in post-migration dietary and physical activity behaviours [16]. According to the theory of planned behaviour, human behaviour is influenced by three main factors: beliefs about the likely results of a behaviour (behavioural beliefs), beliefs about the expectations of significant others (normative beliefs), and beliefs that people can perform a behaviour in the presence of discouraging situations (control beliefs) [16]. Behavioural beliefs produce favourable or unfavourable attitudes towards the behaviour (attitude), normative beliefs often result in behaviours that are supported by significant others (subjective norms), and control beliefs produce the power to perform the behaviour (perceived behavioural control [16]. Together, attitude, subjective norm, and perceived behavioural control lead to the formation of a behavioural intention, and this intention is considered the immediate determinant of behaviour [16].

\section{Study aims}

Examining the association of post-migration dietary and physical activity behaviours with acculturation from an approach that considers the influence by social cognitive factors may be important for developing more effective interventions for populations at risk. Using fat intake, fruits/vegetables/fibre intake, and physical activity among Australian residents born in sub-Saharan Africa as indicators of post-migration weight-related behaviours, this quantitative study examined whether the interplay between weight-related behaviours and acculturation is mediated by attitudes, subjective norm, perceived behavioural control, and intention. Additionally, the study investigated the extent to which the variations in post-migration dietary and physical activity behaviours can be explained by acculturation and social cognitive factors.

\section{Methods \\ Study design}

A cross-sectional community-based survey was conducted in two Australian states: New South Wales (NSW) and Victoria. These two states host the largest population of Australian residents of sub-Saharan African (SSA) ancestry [17]. The survey was administered online and in hard copy.

\section{Ethical considerations}

Ethics approval (Reference Number: HC17018) was received from the Human Research Ethics Committee of the University of New South Wales. The study was conducted in accordance with the National Health and Medical Research Council's (NHMRC) National Statement on Ethical Conduct in Human Research. 


\section{Study participants}

Participants were required to be current residents of Australia, to have resided in Australia for at least 12 continuous months, be born in a SSA country, and to have lived in a SSA country until at least 18 years of age. The study excluded white Australians of SSA ancestry as their food culture is known to be similar to Western food culture [18].

\section{Research settings}

There is a long history of population movement between Africa and Australia [19]. It is known that most Australian residents of African ancestry are concentrated in metropolitan areas, because of the search for job opportunities upon arrival in the country [19]. In his study analysing the history of migration between Africa and Australia, Hugo [19] further highlighted that Sydney and Melbourne, which are the respective capital cities of the states of New South Wales (NSW) and Victoria, are the main metropolis hosting the largest population of Australian residents of SSA ancestry [19]. It is estimated that about 380,000 Australian residents were born in Africa [17], with people emigrating from over forty SSA countries [17].

\section{Participant recruitment}

The study was advertised at African community gatherings (e.g. in churches, mosques, and weddings). Flyers, posters, and word-of-mouth were the main modes of advertisements. To increase awareness of the study among the target population, some African community organisations (e.g. Carers of Africa Inc.) were consulted to assist with promoting the research. Using the social communication platforms (e.g. email, WhatsApp and community meetings) of these organisations, eligible persons were invited to voluntarily participate in the study. Participants were self-selected to ensure the inclusiveness of persons from diverse backgrounds. This self-selected method was considered best for this difficult-to-access population but may not yield a representative sample [1]. To address this limitation, a minimum quota of 50 participants was allotted to each of four distinguished SSA sub-regions of origin: Western Africa, Eastern Africa, Central Africa, and Southern Africa.

\section{Data collection}

Data were collected from November 2017 to September 2018. After pre-testing the survey among ten volunteers, the survey was extended to the target population. The survey was distributed in hard copy and was selfcomplete; however, a researcher was available to provide assistance when required. Interested and eligible persons who could not complete the survey instantly were directed to an online version developed using Qualtrics software. The survey questions focused on retrospective, current, and prospective fat intake, fruits/vegetables/ fibre intake, and physical activity. Of the 304 paper surveys that were distributed, 221 were completed and returned by participants. In addition, 32 surveys were completed online making a total of 253 completed surveys. Based on Cohen's power analysis, a sample size of 253 was deemed sufficient for a significant analysis [20].

\section{Measures}

\section{Acculturation}

To assess acculturation, the Vancouver Index of Acculturation (VIA) was employed [21]. VIA has two subscales, cultural maintenance (heritage dimension) and cultural participation (mainstream dimension). VIA is bi-dimensional and comprises 20 Likert-scale type items $(1=$ disagree to 9 = agree $)$. The Likert-scale type items focused on several domains including cultural values, social relationships, and adherence to tradition. Higher or lower scores on an item indicate that the participant had stronger or weaker connections with either their heritage culture or Australian mainstream culture.

\section{Social cognitive factors}

Measures of the social cognitive factors were developed using recommended guidelines provided by Ajzen [16]. It is important to note that separate social cognitive measures were developed for fat intake, fruits/vegetables/fibre intake, and physical activity. Scores ranged from 1 to 7 , with 1 representing 'the lowest rating on an item', 4 representing 'indifference', and 7 representing 'the highest rating'.

\section{Attitude}

Attitude was measured using six items with each item comprising seven semantic differential scales (i.e. extremely bad-extremely good, extremely useful-extremely useless, extremely unreasonable-extremely reasonable, extremely pleasant-extremely unpleasant, extremely uncomfortable-extremely comfortable, very interestingvery boring). An example of the questions asked about attitude was: "How would you describe it, if you would be eating fruits/vegetables/fibre every day, for the next 3 months?"

\section{Subjective norm}

Subjective norm was measured using three items with seven semantic differential scales (i.e. perfectly correctnot correct at all, strongly agree-strongly disagree, extremely unlikely-extremely likely). An example of a statement about subjective norm was: "Most people who are important to me want me to eat meaty/oily/fatty foods". 


\section{Perceived behavioural control}

Perceived behavioural control was measured using two items with seven semantic differential scales (i.e. definitely true-definitely false, strongly disagree-strongly agree). An example of a statement about perceived behavioural control was: "I am confident that I can do 150 minutes moderate-intensity exercise or 75 minutes vigorous-intensity exercise per week, in the forthcoming month".

\section{Intention}

Intention was measured using two items on a seven semantic differential scale (extremely unlikely-extremely likely, strongly agree-strongly disagree). An example of a statement about intention was: "I plan to do at least 150 minutes moderate-intensity exercise or 75 minutes vigorous-intensity exercise in the forthcoming month".

\section{Dietary behaviour}

Fat intake Block's self-reported fat intake screener was adopted for measuring post-migration consumption of fat and cholesterol [22]. This is a 17-item selfadministered screener that asks participants for frequency of food intake [22]. Frequency of food intake was assessed with the following response options: (a) 1 month or less, (b) 2-3 times a month, (c) 1-2 times a week, (d) 3-4 times a week, and (e) 5 times or more a week [22]. In scoring fat intake, each response option was given a numeric value; 1 month or less $=0 ; 2-3$ times a month $=1 ; 1-2$ times a week $=2 ; 3-4$ times a week $=3$; and 5 times a week or more $=4$ [22]. Scores at or below 7 indicate very low-fat intake (i.e. less than $25 \%$ of energy from fats), scores between 8 and 14 indicate average fat intake (i.e. between 30 and $35 \%$ of energy from fats), scores between 15 and 22 indicate high fat intake (i.e. above $35 \%$ of energy from fats), and scores above 23 indicate very high fat intake (i.e. 40 to $50 \%$ of energy from fats) $[22,23]$.

Fruits, vegetables and fibre intake Block's fruits/vegetables/fibre intake screener was employed to capture post-migration intake of fruits, vegetables, and fibre (fruits/vegetables/fibre) [22]. This is a 9-item selfadministered screener that assesses food intake on a weekly or daily basis. Similar to the fat intake screener, each food item was assigned various response options with associated numeric values: less than 1 week $=0$; once a week $=1 ; 2-3$ times a week $=2$; $4-6$ times a week $=3$; once a day $=4$; and twice or more a day $=5$. Scores below 11 indicate low fruits/vegetables/fibre intake [22].

\section{Physical activity}

To measure physical activity behaviour, version 2 of WHO's Global Physical Activity Questionnaire (GPAQ) was adopted [24]. This comprises 16 questions that capture physical activity undertaken in different behavioural domains: activity at work, travel to and from places, recreational activities, and sedentary behaviour [24]. Total physical activity was calculated as the sum of the total minutes of activity computed for each domain [24].

It is worthy to note that 83 participants with physical activity data were excluded from the calculation of total physical activity based on the strict GPAQ guidelines. For instance, participants who did not provide the number of days of engagement in physical activity at work but provided hours were excluded from the sample to promote accuracy of the findings. Therefore, 170 out of the 253 participants were eligible for consideration in the calculation of total physical activity.

\section{Data analysis}

Internal consistency for all scales was measured using Cronbach's alpha and the values ranged from .71 to .90 as shown in Table 1. Means and standard deviations were computed for acculturation, social cognitive factors, and the dietary and physical activity behaviours. To identify potential mediators in the relationship between post-migration dietary and physical activity behaviours and acculturation, zero-order correlations were also conducted for all the variables.

Hierarchical linear regression was used to assess associations of the dietary and physical activity behaviours with acculturation and social cognitive factors. In the first step, the acculturation variables (cultural maintenance and cultural participation) were entered. In step 2, the social cognitive factors pertaining to the specific behaviours (fat intake, fruits/vegetables/ fibre intake, physical activity) were added to the equation, after acculturation was controlled. After all sets of variables were entered separately for each specific behaviour, the overall model and each independent variable were assessed in terms of the extent to which they explained variance in the dietary and physical activity behaviours.

Checks were conducted to ensure that the data did not violate key assumptions. Multicollinearity tests displayed small correlations $(r<0.5, N=253)$ among participant characteristics; none of the participant characteristics had tolerance $\left(1-r^{2}\right)$ near zero. Outliers, normality, linearity, homoscedasticity, and independence of residuals were inspected in residuals scatterplots as well as normal probability plots, which showed no violation of key assumptions. 
Table 1 Participant characteristics

\begin{tabular}{|c|c|c|c|}
\hline & $a$ & Mean & SD \\
\hline \multicolumn{4}{|l|}{ Acculturation } \\
\hline Cultural maintenance & 0.90 & 7.11 & 1.63 \\
\hline Cultural participation & 0.87 & 5.25 & 1.59 \\
\hline \multicolumn{4}{|l|}{ Attitude } \\
\hline Attitude towards fat intake & 0.86 & 3.06 & 1.29 \\
\hline Attitude towards fruits/vegetables/fibre intake & 0.86 & 4.54 & 1.42 \\
\hline Attitude towards physical activity & 0.80 & 4.24 & 1.25 \\
\hline \multicolumn{4}{|l|}{ Subjective norm } \\
\hline Subjective norm for fat intake & 0.74 & 3.90 & 1.62 \\
\hline $\begin{array}{l}\text { Subjective norm for fruits/vegetables/ } \\
\text { fibre intake }\end{array}$ & 0.71 & 4.76 & 1.62 \\
\hline Subjective norm for physical activity & 0.72 & 4.22 & 1.51 \\
\hline \multicolumn{4}{|l|}{ Perceived behavioural control } \\
\hline Perceived behavioural control for fat intake & 0.75 & 4.72 & 1.48 \\
\hline $\begin{array}{l}\text { Perceived behavioural control for fruits/ } \\
\text { vegetables/fibre intake }\end{array}$ & 0.71 & 4.04 & 1.63 \\
\hline $\begin{array}{l}\text { Perceived behavioural control for } \\
\text { physical activity }\end{array}$ & 0.71 & 4.29 & 1.45 \\
\hline \multicolumn{4}{|l|}{ Intention } \\
\hline Intention for fat intake & 0.71 & 3.50 & 1.54 \\
\hline Intention for fruits/vegetables/fibre intake & 0.76 & 4.29 & 1.62 \\
\hline Intention for physical activity & 0.71 & 4.06 & 1.51 \\
\hline \multicolumn{4}{|l|}{ Dietary and physical activity behaviour } \\
\hline Fat intake & 0.88 & 25.25 & 21.81 \\
\hline Fruits/vegetables/fibre intake & 0.82 & 16.37 & 2.73 \\
\hline Physical activity & - & 178.29 & 176.76 \\
\hline
\end{tabular}

\section{Results}

\section{Participant characteristics}

As shown in Table 1, a mean of $7.11(\mathrm{SD}=1.63)$ was recorded for cultural maintenance whereas cultural participation recorded a mean of $5.25(\mathrm{SD}=1.59)$. With the exception of attitude towards fat intake (mean $=3.06, \mathrm{SD}=$ 1.29 ), subjective norm for fat intake (mean $=3.90, \mathrm{SD}=$ 1.62 ), and intention for fat intake (mean $=3.30, \mathrm{SD}=1.54$ ), none of the social cognitive variables was below the midpoint of 4. Using Block's cut-off as a reference [22], the average fat intake can be described as high in the sample $($ mean $=25.25, \mathrm{SD}=21.81)$. Fruits/vegetables/fibre intake $($ mean $=16.37, \mathrm{SD}=12.73)$ was well above Block's 11 point cut-off [22]. The average amount of physical activity was $178 \mathrm{~min}(\mathrm{SD}=176.76)$ a week, which can be considered as an acceptable physical activity level, according to the standard of the World Health Organization [24].

\section{Mediating role of social cognitive factors}

One of the objectives of this study was to assess whether the relationship between post-migration dietary and physical activity behaviours and acculturation is mediated by social cognitive factors. According to Kenny [25], in a mediation analysis, the independent variable should correlate with the mediator and dependent variables. As can be seen in Table 2, none of the social cognitive factors were in line with Kenny's criteria and, as a result, could not be considered as mediators in the interplay between post-migration dietary and physical activity behaviours and acculturation.

\section{Association of weight-related behaviours with acculturation and social cognitive factors}

Since there was no mediation, analysis that followed focused on the extent to which variations in postmigration dietary and physical activity behaviours were explained by acculturation and social cognitive factors. As can be seen in Table 3, acculturation explained $8 \%$ of the variance in fat intake $(F(2,250)=11.1, p<0.000)$, with cultural participation $(\beta=0.29, t(2,250)=4.71, p$ $<0.000)$ emerging as a significant independent covariate in the equation. The addition of social cognitive factors produced an additional $4 \%$ significant increment in the amount of variance explained in fat intake $\left(R^{2}\right.$ change $=$ $0.04, F(4,246)=5.59, p=0.000)$, with attitude towards fat intake $(\beta=0.12, t(4,246)=2.00, p=0.040)$ emerging as a significant independent covariate in the equation.

Furthermore, acculturation explained $2 \%$ of the variance in fruits/vegetables/fibre intake $(F(2,250)=2.8, p$ $=0.061$ ), with cultural participation emerging as a significant independent covariate in the equation. However, the addition of social cognitive factors did not produce a significant increment in the amount of variance explained in fruits/vegetables/fibre intake $\left(R^{2}\right.$ change $=$ $0.03, F(4,246)=2.82, p=0.061)$.

Table 3 also shows that acculturation significantly explained $4 \%$ of the variance in physical activity $(F(2,167)$ $=3.3, p=0.038)$, with cultural maintenance emerging as a significant independent covariate in the equation. The addition of social cognitive factors produced an additional 5\% significant increment in the amount of variance explained in physical activity $\left(R^{2}\right.$ change $=0.05, F$ $(4,163)=2.56, p=0.022)$, with intention for physical activity $(\beta=-0.17, t(4,246)=-2.08, p=0.039)$ emerging as a significant independent covariate in the equation.

\section{Discussion}

This study sought to examine whether social cognitive factors mediate relationships between post-migration dietary and physical activity behaviours and acculturation among Australian residents born in sub-Saharan Africa (SSA). Additionally, the study aimed to investigate the extent to which acculturation and social cognitive factors explain variations in post-migration dietary and 
Table 2 Associations of dietary and physical activity behaviours with acculturation and social cognitive factors

\begin{tabular}{|c|c|c|c|c|c|}
\hline \multirow[t]{3}{*}{ Participant characteristics } & \multicolumn{2}{|l|}{ Acculturation } & \multicolumn{3}{|c|}{ Dietary and physical activity behaviours } \\
\hline & $\begin{array}{l}\text { Cultural } \\
\text { maintenance }\end{array}$ & $\begin{array}{l}\text { Cultural } \\
\text { participation }\end{array}$ & Fat intake & $\begin{array}{l}\text { Fruits/vegetables/fibre } \\
\text { intake }\end{array}$ & $\begin{array}{l}\text { Physical } \\
\text { activity }\end{array}$ \\
\hline & $r(p$ value $)$ & $r$ ( $p$ value) & $r$ ( $p$ value) & $r$ ( $p$ value) & $r$ ( $p$ value) \\
\hline \multicolumn{6}{|l|}{ Acculturation } \\
\hline Cultural maintenance & & & $\begin{array}{l}0.022 \\
(0.733)\end{array}$ & $-0.004(0.955)$ & $0.194(0.011)$ \\
\hline Cultural participation & & & $\begin{array}{l}0.286 \\
(0.000)\end{array}$ & $0.148(0.018)$ & $\begin{array}{l}-0.020 \\
(0.792)\end{array}$ \\
\hline \multicolumn{6}{|l|}{ Attitude } \\
\hline Attitude towards fat intake & $-0.08(0.222)$ & $0.01(0.886)$ & $\begin{array}{l}0.13 \\
(0.035)\end{array}$ & & \\
\hline Attitude towards fruits/vegetables/fibre intake & $-0.05(0.396)$ & $0.11(0.071)$ & & $0.03(0.600)$ & \\
\hline Attitude towards physical activity & $0.02(0.735)$ & $0.17(0.006)$ & & & $-0.07(0.344)$ \\
\hline \multicolumn{6}{|l|}{ Subjective norm } \\
\hline Subjective norm for fat intake & $0.04(0.498)$ & $0.01(0.947)$ & $\begin{array}{l}0.14 \\
(0.024)\end{array}$ & & \\
\hline Subjective norm for fruits/vegetables/fibre intake & $0.12(0.056)$ & $0.14(0.031)$ & & $0.05(0.430)$ & \\
\hline Subjective norm for physical activity & $0.10(0.129)$ & $0.18(0.004)$ & & & $-0.08(0.309)$ \\
\hline \multicolumn{6}{|l|}{ Perceived behavioural control } \\
\hline Perceived behavioural control for fat intake & $0.03(0.663)$ & $0.08(0.204)$ & $\begin{array}{l}0.05 \\
(0.466)\end{array}$ & & \\
\hline $\begin{array}{l}\text { Perceived behavioural control for fruits/vegetables/ } \\
\text { fibre intake }\end{array}$ & $0.02(0.721)$ & $0.10(0.105)$ & & $0.02(0.763)$ & \\
\hline Perceived behavioural control for physical activity & $-0.09(0.162)$ & $0.16(0.011)$ & & & $\begin{array}{l}-0.142 \\
(0.065)\end{array}$ \\
\hline \multicolumn{6}{|l|}{ Intention } \\
\hline Intention for fat intake & $-0.13(0.837)$ & $-0.03(0.607)$ & $\begin{array}{l}0.09 \\
(0.138)\end{array}$ & & \\
\hline Intention for fruits/vegetables/fibre intake & $-0.01(0.875)$ & $0.15(0.016)$ & & $-0.03(0.655)$ & \\
\hline Intention for physical activity & $-0.01(0.886)$ & $0.15(0.015)$ & & & $-0.20(0.010)$ \\
\hline
\end{tabular}

physical activity behaviours. Contrary to what was hypothesised, the association of post-migration dietary and physical activity behaviours with acculturation was not mediated by social cognitive factors. As also noted in previous studies $[26,27]$, this present finding indicates chances of unmeasured confounding factors. Recognition of confounding factors that affect the potential mediation of social cognitive factors in the interplay between post-migration dietary and physical activity behaviours and acculturation presents a gap in current research. Based on past studies, possible confounders may include acculturation stress [28] and emotions that affect eating behaviour [29].

Consistent with previous studies [30-32], findings from this present study emphasise the significant role played by acculturation in the less healthy dietary and physical activity behaviours that often develop after immigration. More specifically, the significant association between post-migration fat intake and cultural participation in this present study suggests that the study population is likely to consume more 'fatty' foods as they adopt Australia's mainstream culture. In reference to past studies, possible reasons for this present result may include easy accessibility of fast foods in Australia, limited availability of familiar local African foods in Australia, lack of adequate time to prepare local African foods in Australia, and relatively low cost of 'fatty' foods in Australia [1].

There are limited documented studies about subSaharan African culture(s) and physical activity, but the available evidence suggests that local African games, plays, festivals, and dances often involve rigorous physical exercise [33, 34]. As similarly noted in some previous studies [6, 7], findings from this present study suggest that maintenance of these local African cultures that involve physical activity are important for ensuring physical activeness after immigration. There is, therefore, 
Table 3 Hierarchical linear regression for dietary and physical activity behaviours by acculturation and social cognitive factors

\begin{tabular}{|c|c|c|c|c|c|c|c|c|c|}
\hline \multirow[t]{2}{*}{ Step } & \multicolumn{3}{|c|}{ Fat intake $(N=253)$} & \multicolumn{3}{|c|}{ Fruits/vegetables/fibre intake $(N=253)$} & \multicolumn{3}{|c|}{ Physical activity $(N=170)$} \\
\hline & $\beta$ & $t$ & $p$ & $\beta$ & $t$ & $p$ & $\beta$ & $t$ & $p$ \\
\hline \multicolumn{10}{|l|}{ 1. Acculturation } \\
\hline Cultural maintenance & 0.01 & 0.07 & 0.954 & -0.01 & -0.20 & 0.840 & 0.20 & 2.57 & $0.011^{*}$ \\
\hline Cultural participation & 0.29 & 4.71 & $0.000^{* * *}$ & 0.15 & 2.37 & $0.018^{*}$ & -0.03 & -0.42 & 0.672 \\
\hline$R^{2}$ & 0.08 & & & 0.02 & & & 0.04 & & \\
\hline$R^{2}$ change & 0.08 & & & 0.02 & & & 0.04 & & \\
\hline$F_{\text {change }}$ & $11.14^{* *}$ & & & 2.82 & & & $3.35^{*}$ & & \\
\hline \multicolumn{10}{|l|}{ 2. Social cognitive factors } \\
\hline Attitude & 0.12 & 2.00 & $0.040^{*}$ & 0.03 & 0.46 & 0.646 & -0.02 & -0.28 & 0.776 \\
\hline Subjective norm & 0.12 & 1.93 & 0.055 & 0.04 & 0.62 & 0.534 & -0.04 & -0.55 & 0.585 \\
\hline Perceived behavioural control & 0.02 & 0.23 & 0.773 & 0.02 & 0.22 & 0.829 & -0.09 & -1.13 & 0.262 \\
\hline Intention & 0.05 & 0.72 & 0.476 & -0.07 & -1.05 & 0.296 & -0.17 & -2.08 & $0.039^{*}$ \\
\hline$R^{2}$ & 0.12 & & & 0.03 & & & 0.09 & & \\
\hline$R^{2}$ change & 0.04 & & & 0.01 & & & 0.05 & & \\
\hline$F_{\text {change }}$ & $2.67^{*}$ & & & 1.16 & & & $2.10^{*}$ & & \\
\hline
\end{tabular}

a need to promote commonly neglected African cultures that involve physical exercise in this population.

Although the social cognitive factors did not play a significant mediational role in the interplay between the dietary and physical activity behaviours and acculturation, there seems to be some evidence that social cognitive factors contribute to variations in the behaviours. In particular, the significant effect of attitude on post-migration fat intake suggests the presence of unique beliefs about fat intake among participants as attitude is theoretically recognised as a function of a person's behavioural beliefs [35]. In line with a past study [36], high fat intake in Australia may be participants' response to a previously limited accessibility of cheap fatty foods in SSA. High fat intake may also be a deliberate attempt to gain more body weight as postmigration weight gain is thought to be evidence of wellbeing in this population [36].

Furthermore, the significant relationship between post-migration physical activity and intention to exercise indicates that promotion of physical activity in this population may require a deliberate plan. In reference to the intention component of the theory of planned behaviour [16], developing a plan for physical exercise may however be a function of positive beliefs about the benefits of exercising, support from significant others to exercise, and a self-ability to continue with a planned physical exercise in a course of a discouraging event.

Findings also suggest that associations of postmigration dietary and physical activity behaviours with acculturation and social cognitive factors may be contingent on the type of behaviour under examination. The lack of significant associations of post-migration fruits/ vegetables/fibre intake with both acculturation and the social cognitive factors imply that either less change has occurred after immigration in terms of participants' fruits/vegetables/fibre intake or the significant change that occurred is unrelated to acculturation and the social-cognitive factors. To provide a better understanding of this finding, future studies may need to consider a place-comparative approach that tracks migrants' fruits/ vegetables/fibre intake from their countries of birth to the destination countries.

\section{Strengths and limitations}

Although several studies have focused on dietary and physical activity behaviours among African migrants in Australia [1, 6, 7], to the best of our knowledge, this work is among the first to investigate the interplay of post-migration dietary and physical activity behaviours with acculturation and social cognitive factors in this population [1]. While the present study makes an important contribution to knowledge, a number of limitations cannot be overlooked. Caution should be taken when generalising the findings as participants were conveniently sampled. To reduce this sampling limitation, however, participants were recruited from the two Australian states that host the largest population of African migrants, using a range of recruitment strategies. Additionally, self-report measures were used, and this may have resulted in memory and/or social desirability bias. To reduce chances of any reporting bias, the survey was carefully pre-tested and anonymously administered. Participants were also encouraged to skip questions if they 
were not sure of the answers. Regarding dietary behaviour, some local African foods may be missing in Block's fat intake and fruits/vegetables/fibre intake measure. However, the measures adopted for this study have been applied to similar populations, are widely accepted, and had undergone validity and reliability checks [22, 23].

\section{Implication of findings for health promotion}

Research has shown that diet composition and level of physical activity are important modifiable behaviours that can help to reduce the risk of developing weightrelated diseases in several populations [37, 38]. One of the widely recognised and important ways of promoting optimal health across several populations is to encourage control of fat intake [39]. Based on the present findings that attitude and cultural participation were significantly associated with fat intake, it may be useful for health promoters to encourage participants to develop more positive attitudes of adopting less fatty food options in the Australian food market. Moreover, as maintenance of local African culture(s) was significantly related to physical activity, encouraging a continuation of African cultural practises that involve physical activities after immigration may be important for promoting good health in this population.

\section{Conclusions}

This study has demonstrated that acculturation and social-cognitive factors play important roles in the dietary and physical activity behaviours of Australian residents born in sub-Saharan Africa. Findings from this study encourage an extension of current research towards a more holistic approach that considers the influence of social cognitive factors in explaining the relationship between post-migration dietary and physical activity behaviours and acculturation. However, the disqualification of the social cognitive variables as mediators in the interplay between post-migration dietary and physical activity behaviours and acculturation suggests that research of additional variables is needed to provide a better understanding of the processes leading to the behaviours.

\section{Abbreviations}

GPAQ: Global Physical Activity Questionnaire; NCD: Non-communicable diseases; NHMRC: National Health and Medical Research Council; NSW: New South Wales; SD: Standard deviation; SSA: Sub-Saharan Africa; UNSW: University of New South Wales; VIA: Vancouver Index of Acculturation; WHO: World Health Organization

\section{Acknowledgements}

The authors of this paper would like to thank all representatives of the supporting bodies (especially gatekeepers of the religious and community organisations) who assisted with the study advertisement. We also extend our gratitude to all participants for spending time on this study. The authors of this paper acknowledge receipt of funding from the Faculty of Arts and Social Sciences in the University of New South Wales.

\section{Authors' contributions}

IYA conceived the topic; IYA designed the methods; LB, ADA, and JW contributed to designing the methods; IYA collected the data; LB, ADA, JW supervised the data collection; IYA analysed the data; IYA and JW interpreted the data; and LB and ADA critiqued the final work. All authors read and approved the final manuscript.

\section{Funding}

This work was financially supported by the Faculty of Arts and Social Sciences in the University of New South Wales.

\section{Availability of data and materials}

The data supporting this study have been deposited in the OneDrive system of the University of New South Wales. Interested persons might require permission to access the data.

\section{Ethics approval and consent to participate}

Ethics approval (Reference Number: HC17018) was received from the Human Research Ethics Committee of the University of New South Wales (UNSW Sydney). The study was conducted in accordance with the National Health and Medical Research Council's (NHMRC) National Statement on Ethical Conduct in Human Research. Anonymity, privacy, and confidentiality were also ensured during data collection, storage, and reporting.

\section{Competing interests}

The authors declare that they have no competing interests.

\section{Author details}

${ }^{1}$ Centre for Social Research in Health, Faculty of Arts and Social Sciences, University of New South Wales, Sydney 2052, Australia. ${ }^{2}$ School of Public Health and Community Medicine, University of New South Wales, Sydney 2052, Australia. ${ }^{3}$ Present Address: Department of Interdisciplinary Social Science, Utrecht University, Utrecht, Netherlands.

Received: 20 August 2019 Accepted: 10 December 2019

Published online: 21 December 2019

\section{References}

1. Renzaho AMN, Burns C. Post-migration food habits of sub-Saharan African migrants in Victoria: a cross-sectional study. Nutr Diet. 2006;63(2):91-102.

2. Holmboe-Ottesen $\mathrm{G}$, Wandel M. Changes in dietary habits after migration and consequences for health: a focus on South Asians in Europe. Food nutr res. 2012;56. https://doi.org/10.3402/fnr.v56i0.18891.

3. Alyousif Z, Mathews AE. Impact of migration on diet, physical activity, and body weight among international students moving from the gulf countries to the United States. Case Reports Journal. 2018;2(1):1-4.

4. Greenberg H, Deckelbaum RJ. Diet and non-communicable diseases: an urgent need for new paradigms. In: Eggersdorfer MK, Kraemer KB, Cordaro JBM, VA , Fanzo JB, MD) , Gibney MD, Kennedy EB, MA) , et al., editors. Good Nutrition: Perspectives for the 21st Century. Section 2: Nutrition, Health and Economic Status. Basel, Karger 2016. p. 105-118.

5. Western Melbourne Regional Development Australia. African Australian Health in the West 2014. Available from: http://www.health.vic.gov.au/ healthvictoria/nov13/harvest.htm. Accessed 13 Nov 2016

6. Ibe-Lamberts KD. Exploring cultural perspectives of physical activity among transnational African immigrants. Champaign and Urbana: University of Illinois at Urbana-Champaign; 2016.

7. Renzaho AMN, Swinburn B, Burns C. Maintenance of traditional cultural orientation is associated with lower rates of obesity and sedentary behaviours among African migrant children to Australia. Int J Obes (Lond). 2008:32(4):594-600.

8. Berry. Immigration, acculturation, and adaptation. Applied Psychology: An International Review. 1997;46(1):5-68.

9. Nguyen AMD, Benet-Martinez V. Biculturalism unpacked: components, measurement, individual differences, and outcomes. Soc Pers Psychol Compass. 2007;1(1):101-14.

10. Magana JR, dela Rocha O, Amsel J, Magana HA, Fernandez MI, Rulnick S. Revisiting the dimensions of acculturation: Cultural theory and psychometric practice. Hispanic J Behav Sci 1996;18(4):444-468. 
11. Mendoza RH. An empirical scale to measure type and degree of acculturation in Mexican-American adolescents and adults. J Cross-Cult Psychol. 1989;20(4):372-85.

12. Fox M, Thayer Z, Wadhwa PD. Assessment of acculturation in minority health research. Soc sci med. 2017;176:123-32.

13. Norman P, Conner M, Bell R. The theory of planned behaviour and exercise: evidence for the moderating role of past behaviour. Br J Health Psychol. 2000;5(3):249-61.

14. Flay BR, Petraitis J. The theory of triadic influence: a new theory of health behavior with implications for preventive interventions. Adv med soc. 1994:4:19-44

15. Bandura A. Social cognitive theory: an agentive perspective. Annual Review of Psychology. 2001;52:126.

16. Theory of Planned Behaviour Questionnaire. Measurement Instrument Database for the Social Science [Internet]. 2013. Available from: www.midss.ie. Accessed 13 Nov 2016

17. Improved access to historical Census data [Internet]. 2013. Available from: http://www.abs.gov.au/websitedbs/censushome.nsf/home/cowsredirect. Accessed 13 Nov 2016

18. MAZbahr PR. Race and nutrition: an investigation of Black-White differences in health-related nutritional behaviours. Sociology of Health \& IIIness. 2007;29:831-56.

19. Hugo G. Migration between Africa and Australia: a demographic perspective. In Background paper for African Australians: A review of human rights and social inclusion issues. Australian Human Rights Commission.: Sydney NSW; 2009

20. Cohen J. A Power Primer. Psychological Bulletin. 1992;112(1):155-9.

21. Vancouver Index of Acculturation (VIA). Measurement Instrument Database for the Social Science [Internet]. 2013. Available from: http://www.midss.org/ sites/default/files/via.american.pdf. Accessed 13 Nov 2016.

22. Block $\mathrm{G}$, Gillespie C, Rosenbaum EH, Jenson C. A rapid food screener to assess fat and fruit and vegetable intake. American journal of preventive medicine. 2000;18(4):284-8

23. Di Noia J, Schinke SP, Contento IR. Dietary fat intake among urban. African American adolescents. Eating behaviors. 2008;9(2):251-6.

24. World Health Organisation. Global Physical Activity Questionnaire (GPAQ) Geneva, Switzerland: Department of Chronic Diseases and Health Promotion Surveillance and Population-Based Prevention, World Health Organization 2017 [Available from: https://www.who.int/ncds/surveillance/ steps/GPAQ\%20Instrument\%20and\%20Analysis\%20Guide\%20v2.pdf. Accessed 13 Nov 2016

25. Kenny DA. Mediation. 2018. p. http://davidakenny.net/cm/mediate.htm. Accessed 13 Nov 2016.

26. Kley S. Explaining migration as a process of cumulative causation in the life course. Migremus Arbeitspapiere. 2009:1-29.

27. McDermott MS, Oliver M, Svenson A, Simnadis T, Beck EJ, Coltman T, et al. The theory of planned behaviour and discrete food choices: a systematic review and meta-analysis. Int J Behav Nutr Phys Act. 2015;12:162.

28. Tseng M, Fang CY. Stress is associated with unfavorable patterns of dietary intake among female chinese immigrants. Annals of behavioral medicine: a publication of the Society of Behavioral Medicine. 2011;41(3):324-332.

29. Devonport TJ, Nicholls W, Fullerton C. A systematic review of the association between emotions and eating behaviour in normal and overweight adult populations. J Health Psychol. 2019;24(1):3-24.

30. Renzaho AMN. Migrants getting fat in Australia: acculturation and its effects on the nutrition and physical activity of African migrants to developed countries; 2007.

31. Sanou D, O'Reilly E, Ngnie-Teta I, Batal M, Mondain N, Andrew C, et al. Acculturation and nutritional health of immigrants in Canada: a scoping review. J immigr minor health. 2014;16(1):24-34.

32. Onuoha-Obilor SC. 2017 Dietary acculturation and obesity in african immigrant adults. Minneapolis, Minnesota: Walden University; 2017.

33. Amusa $L$, Toriola $A$. The changing phases of Physical education in Africa: can a uniquely African model emerge. Afr J Phys Act Health Sci. 2010;16(4).

34. Ndee HS. Sport, culture and society from an African perspective: a study in historical revisionism. Int J Hist Sport. 1996;13(2):192-202.

35. Conner M, Norman P. Predicting health behaviour. 2nd ed. Buckingham: Open University Press; 2005.

36. Addo IY, Brener L, Asante AD, de Wit J. Socio-cultural beliefs about an ideal body size and implications for risk of excess weight gain after immigration: a study of Australian residents of sub-Saharan African ancestry. Ethnicity \& Health. 2019:1-16.

37. World Health Organisation. Diet, nutrition and the prevention of chronic diseases. Report of the joint WHO/FAO expert consultation, WHO Technical Report Series. Geneva: WHO; 2019.

38. Ford ES, Bergmann MM, Boeing H, Li C, Capewell S. Healthy lifestyle behaviors and all-cause mortality among adults in the United States. Preventive medicine. 2012;55(1):23-7.

39. National Health and Medical Research Council. Australian Dietary Guidelines. In: Ageing DoHa. Canberra: National Health and Medical Research Council; 2013.

\section{Publisher's Note}

Springer Nature remains neutral with regard to jurisdictional claims in published maps and institutional affiliations.
Ready to submit your research? Choose BMC and benefit from:

- fast, convenient online submission

- thorough peer review by experienced researchers in your field

- rapid publication on acceptance

- support for research data, including large and complex data types

- gold Open Access which fosters wider collaboration and increased citations

- maximum visibility for your research: over $100 \mathrm{M}$ website views per year

At $\mathrm{BMC}$, research is always in progress.

Learn more biomedcentral.com/submissions 